\title{
A dilemma that probably would never resolve
}

\author{
Ravi Thanage, Shubham Jain, Sanjay Chandnani, and Pravin Rathi \\ Department of Gastroenterology, Topiwala National Medical College and BYL Nair Charitable Hospital, Mumbai, India
}

Keywords: Hepatitis B virus; Acute hepatitis; Chronic hepatitis

Dear Editor,

Presentation of acute hepatitis $B(A H B)$ and chronic hepatitis $B$ with acute exacerbation (CHB-AE) is clinically identical. The absence of evidence of underlying chronic liver disease (CLD) and history of hepatitis B infection would further worsen differentiation between two conditions. The article by Lall et al.' published in Clinical and Molecular Hepatology brings back the discussion on the table. We appreciate the efforts of the authors for retrospectively compiling baseline virological parameters like immunoglobulin (Ig) M anti-hepatitis B core ( $\mathrm{HBC}$ ) level, hepatitis B virus (HBV) DNA level, quantitative hepatitis B surface antigen, and hepatitis B e antigen values as well as prothrombin time. ${ }^{1}$ However, these findings doesn't add much to the existing literature.

Firstly, out of 83 patients from the CHB-AE group, 60 (72.2\%) were cirrhotic and 23 (27.7\%) were non-cirrhotic. Patient with evidence of underlying CLD the diagnosis of CHB-AE automatically becomes the diagnosis of choice irrespective of the virological parameters.

In day to day practice dilemma arise when there is no clear cut evidence of underlying CLD. Also in the setting of on-going hepatitis, non-invasive diagnostic modalities are likely to yield falsepositive results for cirrhosis. Those with evidence of underlying cirrhosis should have been excluded from the study.
The author reported area under receiver operating characteristic (AUROC) curve for IgM anti-HBc as 0.87. The sensitivity and specificity for the cut-off value of 20.5 signal to cut-off (S/CO) were $93.3 \%$ and $92.7 \%$, respectively, while positive predictive value and negative predictive value at this cut-off were $86.9 \%$ and $95.9 \%$, respectively. However, the use of IgM anti- $\mathrm{HBC}$ has already been well established, many previous studies reported different cut-offs for same. Prospective study from our institute revealed that $76.9 \%$ of patients in the AHB group had high IgM anti-HBC titer ( $>12.14$ $\mathrm{S} / \mathrm{CO})$. On the other hand, low IgM anti-HBC titer ( $<12.14 \mathrm{~S} / \mathrm{CO})$ was seen in the majority (71.4\%) of the patients in the CHB-AE group. ${ }^{2}$ The study by Kumar et al. ${ }^{3}$ have found an incidence of high IgM anti-HBc titer $(>1: 1,000)$ in $77.5 \%$ patients of acute viral hepatitis $B(A V H-B)$ and low lgM anti-HBc titer $(<1: 1,000)$ in 70\% patients of the CHB-AE group. Park et al. ${ }^{4}$ showed cut-off values for IgM anti-HBC as $>8$ S/CO which had sensitivity and specificity of 96.2\% and $89.7 \%$ respectively for diagnosis of $A V H-B$.

In this study, HBV DNA levels were lower in CHB-AE than AHB, the opposite of which was shown in previous studies. A large number of CLD patients in the CHB-AE group probably led to this type of findings. Gayno et al. ${ }^{5}$ have shown that in CHB-AE viral load rises in serum during spontaneous reactivation of chronic hepatitis B infection. In our study, the sensitivity of HBV DNA levels $(>15,390 \mathrm{IU} / \mathrm{mL})$ in the diagnosis of CHB-AE was $78.6 \%{ }^{2}$ The

\section{Abbreviations:}

$A H B$, acute hepatitis $B$; $A \cup R O C$, area under receiver operating characteristic; AVH-B, acute viral hepatitis $B ;$ CHB-AE, chronic hepatitis $B$ with acute exacerbation; CLD, chronic liver disease; $\mathrm{HBC}$, hepatitis B core; $\mathrm{HBV}$, hepatitis $B$ virus; IG, immunoglobulin; INR, international normalized ratio; S/CO, signal to cut-off

\section{Corresponding author : Ravi Thanage}

Department of Gastroenterology, Topiwala National Medical College, 717, 7th floor, OPD Building, Nair Hospital, Mumbai 400008, India

Tel: +91 9028785843, Fax: +91 2223016139

E-mail: ravi.thanage@gmail.com

https://orcid.org/0000-0003-0669-2063 


\section{CUMCALAnd MOLECUAR
HEPPATOLOGY}

study by Kumar et al. ${ }^{3}$ showed high HBV DNA levels $(>0.5 \mathrm{pg} / \mathrm{mL}$ $=28,751 \mathrm{IU} / \mathrm{mL}$ ) had sensitivity and specificity of $86.6 \%, 95.9 \%$ respectively for diagnosis of CHB-AE. Park et al. ${ }^{4}$ showed HBV DNA $<5.5 \log 10 \mathrm{IU} / \mathrm{mL}$ had a sensitivity of $81.1 \%$ and specificity of $72.4 \%$ for the diagnosis of AVH-B.

The author has reported AUROC 0.56 (unsatisfactory diagnostic test) for international normalized ratio (INR). The sensitivity and specificity for the cut-off value of 1.27 were $57.9 \%$ and $45.1 \%$, respectively. INR value could have been affected in the CHB-AE group as many patients had underlying chronic liver disease. The author also reported serum albumin level of $3.2 \pm 0.8 \mathrm{~g} / \mathrm{dL}$ in $A H B$ and $2.9 \pm 0.8 \mathrm{~g} / \mathrm{dL}$ in CHB-AE $(P=0.01)$. The author should have evaluated the diagnostic ability of serum albumin for differentiating these two entities.

To conclude, elderly age, High HBV DNA, and low IgM anti-HBC favors the diagnosis of CHB-AE. Newer biomarkers like HBV RNA, hepatitis $B$ core-related antigen might be useful for differentiating between $A H B$ and CHB-AE in the future.

\section{Authors' contribution}

Ravi Thanage: Manuscript writing

Shubham Jain: Literature search

Sanjay Chandnani: Manuscript writing

Pravin Rathi: Critical revision

\section{Conflicts of Interest}

The authors have no conflicts of interest to disclose.

\section{REFERENCES}

1. Lall S, Agarwala P, Kumar G, Sharma MK, Gupta E. The dilemma of differentiating between acute hepatitis $B$ and chronic hepatitis $B$ with acute exacerbation: is quantitative serology the answer? Clin Mol Hepatol 2020;26:187-195.

2. Thanage R, Rathi P, Pawar V, Udgirkar S, Jain S, Contractor Q, et al. Factors differentiating acute hepatitis $B$ from acute exacerbation of chronic hepatitis B in prospective-retrospective cohort. J Assoc Physicians India 2019;67:39-43.

3. Kumar M, Jain S, Sharma BC, Sarin SK. Differentiating acute hepatitis $B$ from the first episode of symptomatic exacerbation of chronic hepatitis B. Dig Dis Sci 2006;51:594-599.

4. Park JW, Kwak KM, Kim SE, Jang MK, Kim DJ, Lee MS, et al. Differentiation of acute and chronic hepatitis B in IgM anti-HBc positive patients. World J Gastroenterol 2015;21:3953-3959.

5. Gayno S, Marcellin P, Loriot MA, Martinot-Peignoux M, Levy $P$, Erlinger $S$, et al. Detection of serum HBV-DNA by polymerase chain reaction $(P C R)$ in patients before reactivation of chronic hepatitis $B$. J Hepatol 1992;14:357-360. 